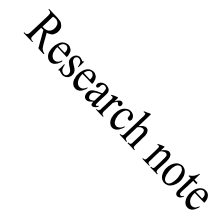

\title{
The energy transfer between a pair of molecules (donor-acceptor) in the vicinity of a graphene-coated nanoparticle
}

\author{
M Jalilian, T Naseri, N Daneshfar \\ Department of Physics, Faculty of Science, Razi University, Kermanshah, Iran \\ E-mail: tnaseri@razi.ac.ir
}

(Received 14 April 2018 ; in final form 20 May 2019)

\begin{abstract}
In this paper, we study the energy transferred between a pair of acceptor-donor molecules near a graphene-coated metallic nanoparticle by using the Laplace equation in a quasi-static approximation. After performing theoretical calculations and computer simulations, the influence of such system parameters as the refractive index of the environment and the contribution of multipolar in different modes on the energy exchanged between the pair of molecules and nanoparticles is analyzed and investigated. The results show that graphene coating enhances plasmonplasmas coupling in the nanostructure, consequently increasing the energy transferred between the two molecules and the nanoparticle. Therefore, this structure could be used as a new route to design nano-based biosensors.
\end{abstract}

Keywords: energy exchanged; acceptor-donor molecule; nanoparticle; graphene

For full article, refer to the Persian section 